\title{
On-line eye movement recorders: The good, the bad, and the ugly
}

\author{
GEOFFREY R. LOFTUS \\ University of Washington, Seattle, Washington 98195
}

\begin{abstract}
The first section of this paper describes three types of problems inherent in the use of on-line eye movement recorders (EMRs). These types of problems are (1) that software is difficult to develop, (2) that an on-line EMR is subject to all difficulties that are normally inherent in computer use and, (3) that with an on-line EMR, it is difficult to detect when the gaze is falling on an object that has an irregularly shaped boundary. It is argued that a large class of eye movement experimentation could be done as easily or more easily with an off-line EMR. The second section of the paper describes an off-line EMR laboratory currently in use by the author.
\end{abstract}

A good deal has been written about eye movement recording systems that are designed to be used on-line with a computer (e.g., Loftus, Mathews, Bell, \& Poltrock, 1975). The principal advantages of on-line eye movement recorders (EMRs) are (1) that they allow collection and analysis of massive amounts of eye movement data in a reasonably short period of time, and (2) that they permit stimuli to be manipulated contingent on various aspects of the eye movement pattern. Few would deny that these advantages are very powerful ones in certain situations.

Unfortunately, however, on-line devices have a number of disadvantages as well. With few exceptions (e.g., Mackworth, 1976), discussion of the disadvantages does not appear in the literature, and the hapless investigator is often left to find out about them for him or herself. A typical syndrome seems to be: 6 months or so after acquisition of a to-be-on-line EMR, the investigator, immersed in implementation problems, realizes that the planned research could be carried out just as easily, or perhaps more easily, with a less sophisticated but also less cantankerous off-line EMR.

The purpose of this paper is twofold. First, I will attempt to enumerate some of the principal disadvantages of an on-line EMR. In the second section, I will describe an alternative off-line system that is currently operating in my laboratory.

\section{PROBLEMS WITH AN ON-LINE EMR}

Three classes of problems are discussed: (1) problems having to do with software development, (2) problems that arise by virtue of yoking an EMR laboratory to a computer, and (3) problems in deciding when specific parts of the visual display are being viewed.

The writing of this paper was supported by National Science Foundation Grant BNS76-23367 to Geoffrey Loftus.

\section{Software}

In principle, the software necessary to compute eye position and to detect saccades on-line is relatively straightforward (cf. Loftus et al., 1975). In practice, however, extensive problems in implementing the software invariably seem to arise. Informal surveys of investigators using on-line EMRs indicate that it generally takes at least 1 year after purchase or construction of the device before any on-line experiments can be run. Even when running begins, the device never seems to be as powerful as had been expected in terms of accuracy and contingent presentation capability. The most serious sorts of software difficulties seem to be the following.

Cross-talk. There is often more horizontal position/ vertical position interdependence than had been anticipated. This difficulty seems to emerge particularly with scleral-reflection devices in which vertical accuracy is highly problematical to begin with. One solution to this dilemma is to use a fairly exotic regression equation in which both vertical and horizontal signals are used as predictors of both vertical and horizontal position. The exact form of the regression equation must depend on the form of the horizontal/vertical interdependence. But, as the equation becomes more complicated, demanding more computer time, timing problems can arise. A second solution is to ignore vertical position, as has been done, for example, by Rayner (1975). Obviously, the second solution is not always practical.

Timing. Several types of on-line EMRs use a TV-scan technique in which eye position can be sampled only once every $17 \mathrm{msec}$. This gives rise to a situation in which detection of saccades can often be quite tricky. (McConkie, Zola, Wolverton, \& Burns, 1978, provide an excellent discussion of this issue.) Other kinds of problems arise when intersampling intervals are shorter. For example, the time needed to execute complicated regression equations as described above can be greater 
than the allotted interval, particularly if such programs are written in a source language such as FORTRAN.

\section{Yoking of an EMR to a Computer}

If an EMR is to be used in conjunction with a computer, it is automatically subject to several problems shared by all on-line experimental devices. First, since computer time is typically limited, use of the on-line EMR is limited by scheduling constrain ts. This problem can be exacerbated because an EMR often demands so much computer time that it cannot be run in a timesharing configuration. Second, computer maintenance time and down time will cut into use of the EMR.

\section{Detection of Fixations on Specific \\ Parts of the Visual Display}

One major use of EMRs is in the investigation of picture perception and picture memory. A question often asked is whether the subject fixated on a specific object during the viewing of the picture (e.g., Loftus \& Mackworth, in press). With a typical on-line EMR, this is not an easy question to deal with. The output from a typical on-line EMR consists of two voltages corresponding to X-and Y-coordinates of the gaze. To determine whether a given $X-Y$ point falls within the boundary of the irregularly shaped area corresponding to some object is a rather difficult programming prospect. Furthermore, the boundary of the object of interest must be somehow read into the computer for each and every object. If the number of such objects is in the hundreds, as in the case of a typical picture-memory experiment, then such a task is rather formidable.

Pilot work and intuitions. A related problem is that, when using an on-line EMR, it is only with difficulty that an investigator can acquire an intuitive view of the nature of the eye movement pattern. Such an intuitive view is particularly helpful when pilot or exploratory work is being done. ${ }^{1}$ A slave scope may be set up with relative ease, thereby permitting the investigator to watch an eye movement pattern in isolation. But, in order to see the eye movement pattern as it falls on the original stimulus, an elaborate projection system or tracing of the stimulus must be utilized in conjunction with the slave scope.

\section{AN OFF-LINE EYE MOVEMENT LABORATORY}

The remainder of this paper describes an off-line, eye movement recording laboratory. The laboratory is relatively inexpensive, involving a total cost of less than $\$ 8,000$. Implementation is simple. Eye movement experiments were being run in our laboratory within 3 days after arrival of the equipment. The laboratory is flexible in a number of respects. Stimulus material consists of anything that can be made into a $35-\mathrm{mm}$ slide, or, if minimal timing accuracy is needed, anything that can be printed on a sheet of paper. When slides are used as stimuli, up to four tachistoscopic shutterequipped projectors can be used in conjunction with each other, essentially producing a four-field tachistoscope. Output from the EMR system is a videotape record consisting of the stimulus being viewed by the subject and a spot of light darting around the stimulus that corresponds to the subject's fixation pattern.

\section{Laboratory Configuration}

Figure 1 shows a typically used configuration of the laboratory. The top panel of Figure 1 is a schematic view; the bottom panel is a photograph of the laboratory. Corresponding numbers in the top and bottom panels mark corresponding components.

As indicated in the top panel, the laboratory can be conveniently divided into a stimulus display system and an eye movement recording system. Indeed, the two
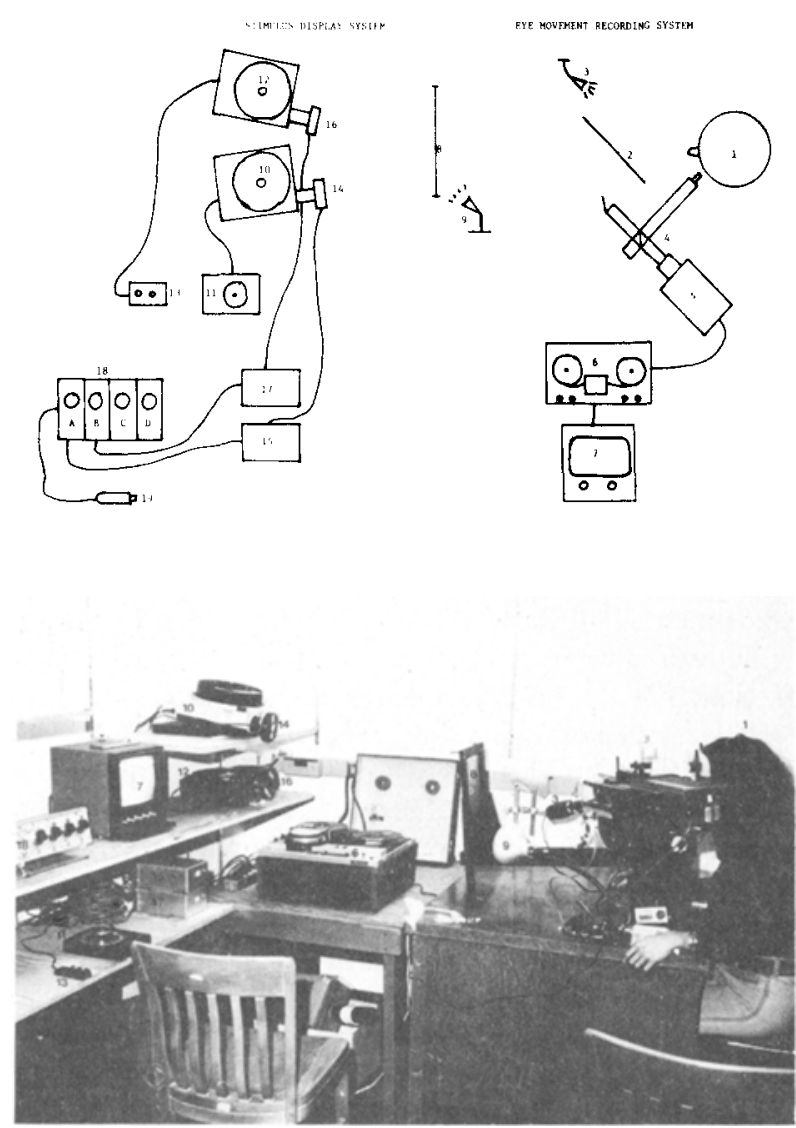

Figure 1. Bottom panel: Photograph of the off-line EMR laboratory. Top panel: Schematic of the off-line EMR laboratory. In both panels, the numbers refer to the following components: (1) subject, (2) half-silvered mirror (beam splitter) (not visible in bottom panel), (3) eye reflection light, (4) Polymetric corneal-reflection EMR, (5) TV camera, (6) videotape recorder, (7) TV monitor, (8) display screen, (9) display screen light, (10) random-access projector, (11) random-access controller, (12) standard Carousel projector, (13) Carousel forward/ reverse controller, $(14,16)$ Gerbrands tachistoscopic shutters, $(15,17)$ shutter power supplies, (18) timer, (19) timer start button. 
systems operate almost completely independently of each other. At the risk of falling into a "neckboneconnected-to-the-hipbone" mode of discourse, I will describe the two systems. In this discussion, numbers in parentheses refer to the numbered components in Figure 1.

Stimulus presentation. A stimulus is always displayed on the display screen (8). It is possible to display material inscribed on a sheet of paper by merely affixing it to the screen. When this is done, a display screen light (9) is necessary to provide sufficient illumination. However, in the more typical display mode, stimuli in the form of slides are back-projected on the display screen via Kodak slide projectors $(10,12)$. In principle, an unlimited number of slide projectors may be used. In my laboratory, which is used primarily for picturerecognition experiments, we generally use two projectors. A random-access projector displays the target picture. A second, standard projector is used to display a masking slide and, when necessary, an eye movement calibration slide.

When the slide projectors are used, stimulus on-off is controlled by Gerbrands tachistoscopic shutters (14, 16). These shutters may be purchased either with or without power supplies. I prefer to purchase them without because power supplies $(15,17)$ can be custom built cheaply (less than $\$ 10$ apiece), and custom-built supplies have several advantages not enjoyed by the commercial versions. Specifically, shutter opening time is fast (less than $1 \mathrm{msec}$ ), and voltage reduction occurs automatically after the shutters open. This allows the shutters to be left open indefinitely without manual adjustment. The power supplies connect to a custombuilt four-in-one set of timers that allows considerable flexibility in the on-off sequence of the shutters.

Eye movement recording. The subject (1) sits in an adjustable-height chair viewing the display screen. The EMR is a Polymetrics corneal-reflection device ("stand camera") described in detail by Mackworth (1967). Briefly, this device operates as follows. The stimulus display is reflected off a half-silvered mirror (2) and thence via another mirror and beam splitter $(4$, the actual EMR) into some optical recording device. Such an optical recording device can be a movie camera, but it is much more convenient to use a closed-circuit TV system. Thus, the stimulus display is recorded by a TV camera (5).

Meanwhile, a reflection light (3) illuminates the subject's left eye. The light is reflected off the cornea and into the EMR (4), where it is columnated, passed through a beam splitter, and eventually guided into the TV camera. When focused, this light takes the form of a small white spot superimposed on the image of the stimulus display. Needless to say, when the EMR is properly calibrated, the position of the recorded dot on the recorded display corresponds to the position of the subject's gaze on the stimulus display.
Table 1

Major Components of the Off-Line EMR Laboratory Shown in Figure 1 and Their Costs (in Dollars) as of 1976

\begin{tabular}{lr}
\hline \multicolumn{1}{c}{ Component } & Price \\
\hline Polymetric 1164 EMR & $\$ 3,710$ \\
Dage MTI-65 W/50 MMF 1.4 Lens & 1,416 \\
Panasonic TR-920V TV Monitor & 197 \\
Sony AV 3650 Videotape Recorder & 1,025 \\
Kodak AV966 RA 960 Random-Access Projector & 861 \\
Kodak Carousel Projector (approximate cost) & 150 \\
Gerbrands G1165 Shutter (cost per shutter) & 175 \\
\hline
\end{tabular}

The TV image is transferred to a videotape recorder (6) and simultaneously to a TV monitor (7), which is positioned so as to be visible to the experimenter but not to the subject.

\section{System Cost}

Table 1 provides a breakdown of the components shown in Figure 1. The only major components not listed are the custom-built shutter power supplies and the custom-built timers. A few comments about several of the components are in order here.

Polymetric EMR. The major problem with this device is that it is highly sensitive to head movements. A bite board is absolutely necessary; even with the bite board, adjustment is necessary on the order of once every $10-30 \mathrm{sec}$. (The variation in the interadjustment interval is due primarily to the "jitteriness" of the subject.) Such an adjustment typically takes about $2-3 \mathrm{sec}$ for an experienced experimenter.

Dage MTI-65 TV camera. The principal requirement of any TV camera to be used in this system is that it be sensitive to the low light levels emanating from the EMR. The low light levels stem primarily from the fact that the light corresponding to the stimulus display must pass through two beam splitters en route from the projector to the camera. This requirement necessitated a rather thorough search for an adequate camera, and the Dage MTI.65 turned out to be ideal.

Sony AV3650 videotape recorder. A crucial requirement for a videotape recorder is that it be capable of playback at an arbitrarily slow speed for eye movement analysis. A recording-to-playback ratio of approximately $1: 10$ is quite suitable for most types of eye movement data analysis. Specifically, both $X \cdot Y$ position and fixation durations (derived from the 60-cycle scan sweeps) can be easily recorded at this playback speed.

\section{CONCLUSIONS}

The system just described operates completely offline and is therefore not privy to the fast analysis and contingent presentation mode of which a sophisticated on-line system is capable. But, by the same token, it is not subject to the rather formidable and demoralizing difficulties enumerated earlier. The system is limited 
with respect to the data it can be used to collect, but it turns out to be suitable for a rather substantial amount of eye movement research. In my own work, I find that it makes a good deal of sense to carefully distinguish between research that requires a sophisticated system vs. research that does not. Or, expressed another way, if I must travel from Seattle to San Antonio, a 747 is my preferred means of transportation. But for routine, garden-variety travel such as from my house to my office, a 747 is more trouble than it is worth and a bicycle does just fine.

\section{REFERENCES}

Just, M., \& Carpenter, P. Eye fixations and cognitive processes. Cognitive Psychology, 1976, 8, 441-480.

LofTUs, G., \& MACKWORTH, N. Cognitive determinants of fixation location during picture viewing. Journal of Experimental Psychology: Human Perception and Performance, in press.

Loftus, G., Mathews, P., Bell, S., \& Poltrock, S. General sottware for an on-line eye-movement recording system. Behavior Research Methods \& Instrumentation, 1975, 7, 201-204.

MackWorth, N. A stand camera for line-of-sight recording. Perception \& Psychophysics, 1967, 2, 119-127.

Mackworth, N. Ways of recording line of sight. In R. A. Monty \& J. W. Sanders (Eds.), Eye movements and psychological processes. Hillsdale, N.J: Erlbaum, 1976.

McConkie, G., Zola, D., Wolverton, G., \& Burns, D. Eye movement contingent display control in studying reading. Behavior Research Methods \& Instrumentation, 1978, 10, 154-166.

RAYNER, K. The perceptual span and peripheral cues in reading. Cognitive Psychology, 1975, 7, 65-81.

\section{NOTE}

1. As an example of this phenomenon, Just and Carpenter (1976) reported an experiment involving an eye movement analysis of the Shepard/Metzler "mental rotation" task. The origins of that experiment consisted of $30 \mathrm{~min}$ worth of "I wonder what would happen if . .." pilot work using the sort of off-line device described in the next section. The pilot work was carried out on a whim, in large part because it was so easy to do and yielded such immediate, intuitively interpretable data. 